\title{
Kritik der symbolischen Formen I - Symbolische Form und Funktion
}

\author{
Peter Dirksmeier \\ Humboldt-Universität zu Berlin, Geographisches Institut, Unter den Linden 6, 10099 Berlin, Germany \\ Correspondence to: Peter Dirksmeier (peter.dirksmeier@geo.hu-berlin.de)
}

Published: 18 March 2016

Steineck, R. C.: Kritik der symbolischen Formen I - Symbolische Form und Funktion, Philosophie Interkulturell Band 3, Fromann-Holzboog, Stuttgart, Bad Cannstatt, 148 S., ISBN-13: 978-3-7728-2673-3, EUR 38, 2014.

Das von dem Züricher Japanologen Raji C. Steineck vorgelegte Werk über die Philosophie der symbolischen Formen von Ernst Cassirer nimmt sich einer wichtigen und interdisziplinär bedeutsamen Aufgabe an. Das Ziel der kurzen aber ausgesprochen gehaltvollen Schrift ist es, ,in Auseinandersetzung mit japanischen Quellen die von Ernst Cassirer vorgeschlagene ,Philosophie der symbolischen Formen" methodisch wie inhaltlich weiterzuentwickeln" (S. 1), wie der Autor bereits im ersten Satz betont. Es versteht sich von selbst, dass ein solch komplexes Ansinnen nicht in einer knapp hundertfünfzigseitigen Schrift zu bewerkstelligen ist. Daher ist das Projekt mehrbändig angelegt. Der vorliegende erste Band hat in diesem Zusammenhang die Aufgabe, die knapp hundert Jahre alten Gedanken und Argumente Cassirers zunächst grundlegend $\mathrm{zu}$ ergänzen und zu modifizieren, sie mit dem aktuellen Stand der Kulturphilosophie zu versöhnen, um sie dann anschließend in den folgenden Bänden kulturtheoretisch weiter zu führen. An dieser Stelle erkennt der Autor einen Bedarf in der Wissenschaftslandschaft, da der Begriff und die Vorstellung von Kultur im wissenschaftlichen Sinne im 19. und frühen 20. Jahrhundert bisweilen lediglich zur Verdeutlichung europäischer Überlegenheit erforscht und beforscht wurde. Dieser, in Teilen der Kulturtheorie wie Kulturphilosophie eingelagerte Eurozentrismus bedarf rückwirkend eines Gegengewichts. Die Philosophie der symbolischen Formen, obwohl in dieser eher eurozentristischen Zeit entstanden, weist für Steineck das Potenzial auf, dieses Gewicht darzustellen.

Wesentlich für Cassirers Hauptwerk ist das Ausloten der Mannigfaltigkeit objektiver symbolischer Ausprägungen im
Zusammenhang mit der Einheit des subjektiven menschlichen Geistes. Die Philosophie der symbolischen Formen lässt sich als die Bündelung der Vielfalt menschlicher Anschauungen in ein bedeutungskonstituierendes Modell auffassen, das sämtliche Formen der sinnlichen Wahrnehmung in sich vereint. Die Kontrastfolie, an der die Argumente Cassirers von Steineck in ihrer Geltung geprüft werden, ist die Philosophie Japans. Anhand der Lektüre japanischer philosophischer Quellen erfolgen die Weiterentwicklung der cassirerschen Gedanken und die Dezentrierung der impliziten eurozentrischen Abweichung. Mit dieser intellektuellen Bewegung wird ein deutlicher Schritt in Richtung einer Kulturtheorie unternommen, die nicht länger eine europäische Kulturtheorie ist. Hier liegt das große Potenzial der Studie für die Humangeographie verborgen: Eine vom Eurozentrismus bereinigte Kulturtheorie cassirerscher Provenienz kann die Kulturgeographie nach dem Cultural Turn bereichern und etwa postkolonialistische Ansätze mit einer Kulturauffassung versorgen, die in ihrem Gestus weder universalistisch noch eurozentristisch ist, genauso wie sie unmittelbare Bezüge zur humanistischen Geographie, etwa den Arbeiten von Nicholas J. Entrikin oder Yi-Fu Tuan, herzustellen in der Lage wäre.

Steineck kritisiert die Kulturauffassung von Cassirer aufgrund ihrer implizierten teleologischen Grundstruktur, die sich anhand der Konzipierung der symbolischen Formen zeigt. Die symbolischen Formen lassen sich in einer hierarchisch geordneten Reihe denken mit dem Mythos auf der untersten Stufe, gefolgt von der Sprache und ihren mannigfaltigen Differenzierungsmöglichkeiten bis hin zur modernen Naturwissenschaft als die höchste und komplexeste Form der symbolischen Abstraktion und menschlichen Kultur. Steineck erscheint eine solche Ordnung, die er anhand eines $\mathrm{Zi}$ tates aus Cassirers „An Essay on Man“ verdeutlicht, als zu einfach. Cassirer übersähe darüber hinaus die gesellschaftli- 
che Rückgebundenheit der Wissenschaft, die Cassirer selbst nicht beobachtet und die heute ein wichtiges Themenfeld der Science Studies darstellt, die wiederum ebenfalls Resonanz in der Humangeographie findet. Steineck sieht es allerdings als Leistung von Cassirer an, die paradoxe Situation adressiert zu haben, dass die Methodik der Wissenschaften eine freie, jedoch nicht uneingeschränkte Form des Handelns voraussetze, die erst wissenschaftlich zu nennen wäre, und die dennoch über das zu entwickelnde wissenschaftliche Erklärungsmodell wiederum reduziert werden solle.

In der Lesart der symbolischen Formen von Steineck zeigt sich ein für die Humangeographie nach dem Cultural Turn bedeutsames Postulat, das der Zürcher Japanologe folgendermaßen formuliert. Die Philosophie der symbolischen Formen „,unterläuft [...] Gegensätze wie Realismus versus Relativismus oder Materialismus versus Idealismus durch einen eigentlich ganz einfachen Hinweis: Was immer von diesen Positionen behauptet und bewertet wird, ist, genau wie diese Positionen selbst, etwas symbolisch Vermitteltes. [...] Erstens, es bietet keinen archimedischen Punkt außerhalb der Welt der Zeichen, und zweitens, es kann selbst nur gebildet werden durch die Ausrichtung an und Akzeptanz von bestimmten Normen“ (S. 69). Ein solches Nachdenken über die Rolle der Normen in einer symbolisch vermittelten Welt mithilfe der Philosophie der symbolischen Formen könnte spannende Wege auch in Arbeiten der Neuen Kulturgeographie aufzeigen und alternative Formen der Analyse der Welt der Zeichen öffnen, die wiederum dem Menschen etwas über die symbolisch vermittelte Welt sagen. Steinecks Weg, über japanische Philosophie solche Querbezüge herauszuarbeiten, macht die Studie in besonderer Weise lesenswert.

Ein humangeographisch interessanter Aspekt an Cassirers Philosophie ist unter anderem sein Nachdenken über den Symbolbegriff. Im Symbol drückt sich eine Beziehung von Sinn und Sinnlichkeit als Ideelles und Materielles auf der Basis einer unterscheidenden Relation aus. Das Symbol ist „ein Gegebenes, durch das in eins gesetzt werden (i) eine Unterscheidung zwischen ihm selbst und einem Anderen und (ii) eine Beziehung in der Weise, dass das Symbol für dieses von ihm unterschiedene Andere steht“" (S. 29). Steineck nimmt nun eine Ergänzung an diesem wichtigen Punkt der cassirerschen Symbolphilosophie vor. Der Zürcher Japanologe erlaubt die Inklusion des Anderen in das Symbol, damit Situationen, in denen das Zeichen erst ,die Anwesenheit des Bezeichneten" (S. 29) konstituiert, gleichfalls von der Theorie erfasst werden können. Steineck führt als Beispiel die Synekdoche an, bei der ein Teil für das Ganze und das Ganze nur für einen Teil stehen kann. Der ursprüngliche Symbolbegriff der Philosophie der symbolischen Formen würde diesen Aspekt ausschließen, so Steineck. Symbolische Verhältnisse sind ein wichtiger Teil humangeographischen Arbeitens. Man denke nur an die Landschaftsanalysen von Stephen Daniels und Denis Cosgrove und beispielsweise deren Arbeiten über die symbolischen Systeme von englischen Landschaften des 18. Jahrhunderts, die bereits auf Cassirer (und Er- win Panofsky) Bezug nehmen. Steinecks Analyse greift damit indirekt ebenfalls auf ein Thema zurück, das am Beginn der Neuen Kulturgeographie steht. Es könnte daher besonders für Humangeograph/innen, die an Repräsentationen und Symbolsystemen interessiert sind, lohnenswert sein, die Studie von Steineck zu lesen.

Sehr verdienstvoll ist die am Ende auf den Seiten 138 bis 140 präsentierte sehr verständliche Darlegung der wichtigsten Aspekte der Philosophie der symbolischen Formen in 14 methodischen Grundsätzen. Diese Form der Zusammenfassung von Cassirers Hauptwerk bietet auch Leser/innen einen unmittelbaren Zugang zu Cassirers Gedankenwelt, die sich bis dato noch nicht tiefgreifend mit den Arbeiten des Philosophen auseinandergesetzt haben. Steinecks Kritik an Cassirer erscheint dabei sehr plausibel, der vernachlässigte Aspekt gesellschaftlicher Konnektivität der in der Philosophie der symbolischen Formen dargelegten symbolischen Mechanismen, und die Antworten, die Steineck noch in Auseinandersetzung mit japanischen Quellen erarbeiten wird, lassen Hilfreiches auch für die Kulturgeographie erahnen. Die ,philosophische Kritik der Kultur“" (S. 134), die dann im Zentrum stehen soll, ist im Kontext von Nationalismus, religiösen Fundamentalismus und weiteren Formen ideologischen Totalitarismus als ausgesprochen notwendig anzusehen. Steineck gelingt mit dem vorliegenden Band ein wichtiger Schritt in diese Richtung, der sicherlich weit über die Grenzen der Philosophie hinaus Beachtung erfahren wird. 